CYBERNETICS AND INFORMATION TECHNOLOGIES • Volume 16, No 5

Special Issue on Application of Advanced Computing and Simulation in Information Systems

Sofia $\bullet 2016$

\title{
Research on the Image Denoising Method Based on Partial Differential Equations
}

\section{Xiaolu Xie}

\author{
Media Art College, Chongqing College of Electronic Engineering, Chongqing 401331, China \\ Email:273999573@qq.com
}

\begin{abstract}
In this paper we propose a new approach for image denoising based on the combination of PM model, isotropic diffusion model, and TV model. To emphasize the superiority of the proposed model, we have used the Structural Similarity Index Measure (SSIM) and Peak Signal to Noise Ratio (PSNR) as the subjective criterion. Numerical experiments with different images show that our algorithm has the highest PSNR and SSIM, as well as the best visual quality among the six algorithms. Experimental results confirm the high performance of the proposed model compared with some well-known algorithms. In a word, the new model outperforms the mentioned three well known algorithms in reducing the Gibbs-type artifacts, edges blurring, and the block effect, simultaneously.
\end{abstract}

Keywords: Image denoising method, Partial differential equations, The blending algorithm.

\section{Introduction}

An image is usually corrupted by noise in its acquisition and transmission. The degraded image severely affects the following image processing, such as image super resolution, image segmentation, image recognition, feature extraction. Thus, image denoising becomes a fundamental and important image processing for improving the quality of an image. The goal of image denoising is to get a clearer and richer detailed image. Preserving the important structures, such as edges and textures has important theoretical significance and application values. It is wellknown that image structures are important for visual perception. Hence, paper Li u et al. [1] mainly focuses on image denoising model for edge-preservation and texture-preservation. Several new image denoising methods are proposed based on wavelet transform, kernel regression and nonlocal means, respectively. The main achievements and innovations are as follows: Using the scale correlation and maxima modulus of wavelet coefficients, Liu define two new wavelet correlation coefficients based on maxima child nodes, and propose a new scale correlation based image denoising method. Furthermore; Liu extend these definitions to 
fractional B-spine wavelet coefficients. Both theoretical and experimental results demonstrate that the proposed correlation coefficients can capture the structure information in high frequency sub bands. So more edges and textures are kept in the denoised image and the quality of denoised image is improved.

To overcome the shortcomings of the regularity exponent based image denoising model, a new image denoising model combing the regularity exponent and image Total Variation (TV) is proposed. The model fully utilizes the relationship between wavelet coefficients and signal regularity. So the image regularity is modified by changing the wavelet coefficients in different scales. The noise is reduced, while sharp edges are preserved; meanwhile, the Gibbs phenomenon is disappeared. With the fractional B-spline wavelet instead of the traditional wavelet, Liu establish a denoising algorithm based on the fractional B-spline wavelet and total variation. In this case, edges and textures are both maintained in the denoised image [3]. An adaptive kernel regression model based on structure tensor is proposed. The structure tensor, which can exploit the local gradient structure information, provides information to achieve a data-adaptive kernel function. Due to the accurate estimation of edge orientations, edges and textures structure information are well preserved and a better visual effects are achieved during denoising and interpolation. Meanwhile, the RMSE also proved the effectiveness of the algorithm. To overcome the shortcomings of Steering kernel regression, two more robust kernel functions are applied to kernel regression, which are robust to micro-edges. On the image edges, the kernel functions have a faster decay, and the weight of pixels are assigned a small value. Thus it discourages the pseudo-edges. Experimental results of image denoising and interpolation show that the efficiency of the proposed models, especially to the image with less textures and more edge details. In $\mathrm{Ca} \mathrm{i}$ and $\mathrm{W}$ e i [4] and [5-7] is proposed a novel nonlocal TV variation model, where the fidelity term is based on the Patch similarity, and regularity term is nonlocal TV priori. The iterative nonlocal provides structure similarity between noisy and denoised images; while the nonlocal TV preserves the edge and texture details. Compared with other related denoising methods, the proposed model can preserve more structure information in denoised image, especially to the image with much noise. Combining the nonlocal Patch similarity regularization with $\mathrm{TV}$ regularization, $\mathrm{Fan} \mathrm{g}$ and $\mathrm{C}$ a o [8] proposes a new nonlocal Patch self-similarity regularized image denoising model. The similarity of Patches are said to be accurate, by introducing adaptive structure tensor to compute weight function of nonlocal Patch similarity. So in the denoised image, more structural features can be retained. A simpler and more effective algorithm, Split Bregman algorithm, is used to solve the model iteratively. By extending the model to image restoration, our model improves the quality of restoration image and the efficiency of computational complexity.

\section{Introductions to Image denoising method}

With the development of digital image processing techniques, it's applied more and more extensively. Due to the influence of factors by noise in the process of 
acquisition and transmission, it makes the degradation of the quality of images. The noise characteristics of image and its recovery is one of the hot issues of the imageprocessing technology, the study of image denoising can help the subsequent work of image edge extraction and it's also the basic of the subsequent image processing. The image edge contains a lot of information; it has significant effect on image segmentation and recognition by using the edge detection effectively and accurately. Therefore, it has the vital significance to research on the image denoising and edge detection. Paper Y in, Che n and Li [9] analyzes the current image denoising and edge detection processing technology, combined the algorithm of image denoising and edge detection in the aspect of airspace, and then presented some algorithms, through the theoretical analysis and experimental results, it proved that such algorithms are effective and feasible. The main results in paper of Y an and Sui [3] are: the scanning image denoising based on the Otsu method Scanning is one of the important ways to obtain image, while the image through scanning often remain shadows, it seriously affected the quality of the scanning images. Calculate the histogram using in the scanning images and the appeared probability of all the grayscale. According to the histogram used in the scanning images have the features with bimodal, through the probability of the grayscale and the Otsu's method, to gain the best threshold, and then use the threshold value to distinguish the foreground images and the background shadows, in order to realize the removing for the shadows of the scanning images. It has already achieved a good effect and a lot of experimental results have demonstrated the effectiveness of the proposed method. An edge-preserving image denoising method Pulse noise is the common types of image noise. Paper [3] studied the method to remove the pulse noise of the images, and firstly use the convolution kernel in four directions to calculate the good pixels and noise pixels, then retain the information of the good pixels. As for the noise pixels in the $3 \times 3$ adjacent domain, find out the minimum distance of the good pixels, if there is nothing but the good pixels, the adjacent domain will expand for $5 \times 5$, then it needs to search the good pixels; if the good pixels exist, replace the mid-value with the noise pixels, otherwise replace the farthest distance pixel values in the adjacent domain with noise pixels. The experimental results shows that the algorithm can not only remove the image noise effectively, but also can better protect the edge of the image. The image edge detection has the value for widespread practical application, paper [3] researched on the multi-peak image edge extraction algorithm, and firstly, through all the gray scale expectations to confirm the area of the image segmentation thresholds, using the maximum variance (Otsu) method combined with the expectation to calculate the image segmentation threshold, using the morphological method to eliminate isolated points and small cavities for the image that has been segmented. Finally, using Sobel operators to undergo the edge detection respectively for the segmented image and the original image, adding the two edge images can be obtained the result of the edge detection. Experiments show that this algorithm less of missing inspection and accurate positioning the image edge. The image edge detection is based on the morphology and enhancement algorithm. The morphology is a simple and effective method in the area of image processing. Due to poor effect on the 
weak edge of the edge images detected by morphology, paper [3] detected that the edge images have characteristics of single-peak, which is based on the morphologic, and found out the minimum and maximum values in the histograms. Through these two point,s available a straight line, then all points to a maximum distance of this line are found out, using the largest point corresponding the gray scale as the segmentation thresholds; and then use binary threshold to achieve the purpose of enhanced the contrast between the poor effect on the edge images and the edge images. Lots of experiments show that this algorithm can solve the poor effects on the weak edge, so the method of enhancing the edge image can accurately reflects the original image edge information.

Efficient sparse representation and processing of unstable signal are the main contents in mathematics and information science. Recently, the Discrete Wavelet Transform (DWT) has become efficient in the sparse representation of unstable signal and also is a powerful tool for signal and image processing. It, however, has some disadvantages, including, (1) it is shift sensitive because the input signal shift generates unpredictable changes in DWT coefficients; (2) it suffers from poor directionality because DWT coefficients reveal merely three spatial orientations; (3) it lacks of the phase information that accurately describes non-stationary signal behavior; that undermine its usage in many applications. Therefore, there is a strong motivation to study new types of wavelet transforms with better shift invariance and directionality. Due to the imperfection of image acquisition systems and transmission channels, the observed images are often in low-quality or degraded by noise. The goal of image denoising is to remove the noise while retaining as much as possible the important features (edges) and obtain acceptable image for vision. The image enhancement algorithms are to process a given image so the results are better than original image for their applications or objectives. Noise elimination and image enhancement are still the most fundamental, widely studied, and largely unsolved problems in computer vision and image processing. To overcome the disadvantages of the traditional DWT, this thesis mainly focus on two new types of complex wavelet transform: the Dual-Tree Complex Wavelet Transform (DT-CWT) and the higher density dual tree DWT. The properties of the DT-CWT such as approximate shift-invariance, directionality and implementation issue are carefully investigated. Furthermore, a new algorithm to construct wavelet filter bank of the DT-CWT is presented. At the same time, a new complex wavelet transform - the higher density dual tree DWT is introduced and the corresponding characteristics are studies and a design procedure to obtain Finite Impulse Response (FIR) filters that satisfy the numerous constraints imposed is developed. To better process the non-stationed signal, the total variation and optimization based schemes for signal and image adaptive decomposition are preliminarily studied. Some classical applications of the proposed complex wavelet transforms are also further studies such as image denoising and enhancement. Results of experiments show that the proposed new algorithms perform better than the existing methods at present. The main achievements in Yin's paper are as follows: first, an approach for designing orthogonal DT-CWT filters is proposed; where the two related wavelets pairs form approximate Hilbert transform pairs. Different from the existing design 
techniques, the two wavelet filter banks obtained here are both of linear phases. By adjusting the parameters, wavelet filters with rational coefficients may be achieved, which can speed up the DT-CWT effectively. The transform corresponds to a new family of dyadic wavelet tight frames based on two scaling functions and four distinct wavelets. This design procedure employs a fractional-delay all pass filters, spectral factorization and the solutions have vanishing moments, compact support, a high degree of smoothness, intermediate scales, approximate Hilbert transform properties, and are nearly shift-invariant. Experimental results show that the proposed methods are effective to a wide range of signals and images; when compared to the fixed wavelet bases method, the produced reconstruct images with our adaptive method are with better PSNR and visual quality. Based on the theory above, to address the problems of the image denoising and enhancement, the image denosing and enhancement in the new types of complex wavelet transform domain in detail is proposed. Three new image denoising algorithms based on the DT-CWT are proposed: (i) a new locally adaptive image denoising method, which exploits the intra-scale and inter-scale dependencies in the DT-CWT domain; (ii) a new nontraining Complex wavelet Hidden Markov Tree (CHMT) model, which is based on the DT-CWT and a fast parameters estimation technique; (iii) a new denoising algorithm based on the Gaussian Scale Mixture (GSM) of the coefficients of the DT-CWT. These methods exploit the properties of the DT-CWT and the statistics of the coefficients and the obtained better denoising performances while reducing the computational complexity. At the same time, we introduce an effective integration of the intrastate correlations within the interstate SURE based orthonormal wavelet thresholding, which can solve the problem of the interstate method that is not very effective for those images that have substantial highfrequency contents. In addition, we also investigate the image enhancement based on the new types of wavelet transform and the statistical characters of visual representation and propose two new method of image enhancement: (i) a novel method for image enhancement, which exploits the properties of the double-density dual-tree DWT and the statistical characters of visual representation; (ii) a new method for noisy image enhancement, which is based on the GSM model of the DT-CWT coefficients and the combination of the DT-CWT and the statistical characters of visual representation, and can optimize the contrast of image features of while minimizing image denoise.

\section{Partial differential equations}

Image processing has been an active field of research resulting from the development of computer technology and increasing requirements of multimedia data processing. Since image denoising and enhancement are often applied to improve the image quality and to make them fulfill the specific application requirements, both of them are important research subjects in image processing. In this paper, more attention is paid to the partial differential equation based methods, especially to the theoretical and application difficulties in these methods. With the topics of image denoising and enhancement, we have reviewed and studied the 
following aspects in this thesis: the PDEs based on scale space theory, variation PDEs and geometrical PDEs based on curve or surface evolution. The difficulties in them are analyzed and solutions to them are proposed.

PM model and ID model have good performance in the flat areas, and TV model has good performance in the edges. Hence in our present work the gradient in each region of the image is calculated as a measure of the amount of image details.

During the processing of the image smoothing by our scheme, the image gradient keeps changing as the iterative evolution changes. For this reason, the threshold, which is used to determine whether the region is the edge or the flat area, should not be set fixed, but keeps changing with the number of iterations.

To restore the original image $u$ by the degraded image $u_{0}$, we take the energy function of the image as follows:

$$
\left.E(u)=\iint_{\Omega}^{\left[\phi|\nabla u|+\beta|\nabla u|^{2}+\delta k^{2},\right.} \operatorname{In}\left(\frac{1}{g(|\nabla u|)}\right)+\lambda\left(u-u_{0}\right)^{2}\right] d x d y,
$$

Here

$$
\begin{gathered}
k=k_{0} e^{-\Delta t(n-1)}, \\
g(|\nabla u|)=\frac{1}{1+\left(\frac{|\nabla u|}{k}\right)^{2},} \\
\mu=e^{\frac{-1}{|\nabla u| / L}}, \\
\left\{\begin{array}{l}
\phi \\
\beta=-4 \mu\left(\mu-\frac{1}{2}\right), \\
\delta=2(\mu-1 / 2)(\mu-1) .
\end{array}\right.
\end{gathered}
$$

From the new model we can expect that:

1. In the region which contains more image features (such as edges, etc.), the new model will play good role to preserve the edges of the image, namely this model will highlight the Total Variation (TV) model, therefore $\mu$ should be close to one.

2. In the flat areas of image, which contains less image features, and less noise, the model will highlight the role of the PM model; therefore $\mu$ should be close to zero.

3. In the flat areas of image, which contains less image features, and much noise, the new model will highlight the roles of the ID model; therefore $\mu$ should be close to 0.5 .

To solve problem by using the finite difference method, we have equations: 


$$
\begin{gathered}
P=\nabla \cdot(g(|\nabla u|) \nabla u), \\
T=\nabla \cdot\left(\frac{\nabla u}{|\nabla u|}\right), \\
S=\nabla \cdot(\nabla u) .
\end{gathered}
$$

\section{Experimental results and analysis}

In this section we compare the proposed approach with other methods in terms of the visual quality of denoising image, Structural Similarity Index Measure (SSIM), and Peak Signal to Noise Ratio (PSNR) according to the equations (1)-(8).

In Figs 1, 2, 3 the original images are corrupted by salt and pepper noise, white Gaussian noise and speckle noise respectively The experimental results are shown in Figs 1, 2, 3, where in each figure is illustrated respectively the results by the ID model, the PM model, the TV model, the W model, the WT model and our new model.

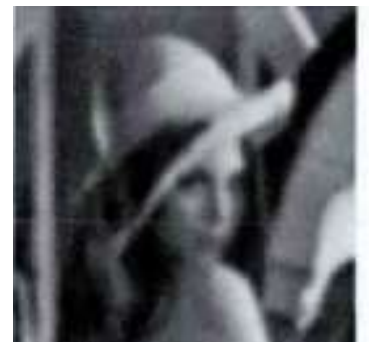

(a)

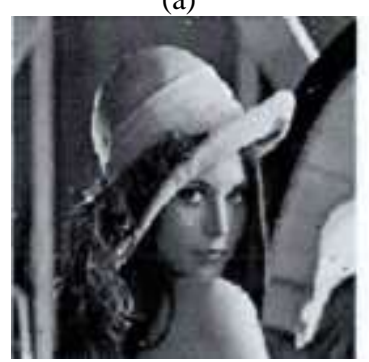

(c)

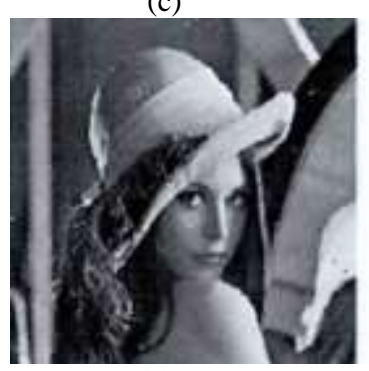

(e)

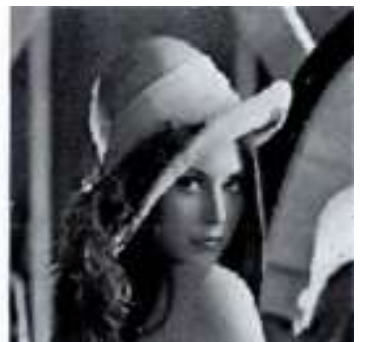

(b)

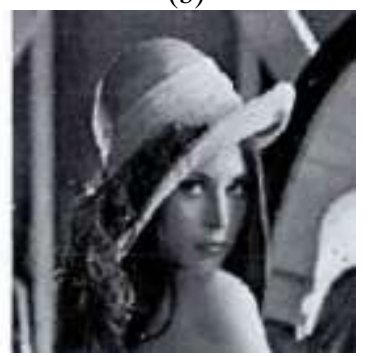

(d)

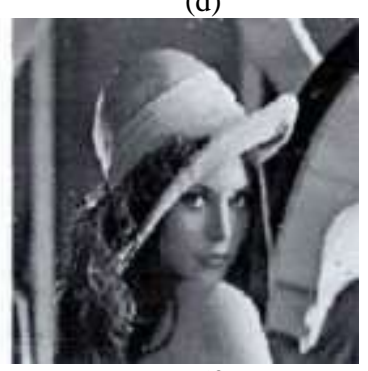

(f)

Fig. 1. Results of denoising obtained with Lena image: Result of ID algorithm (a); result of PM algorithm (b); result of TV algorithm (c); result of W algorithm (d); result of WT algorithm (e); result of new algorithm (f) 


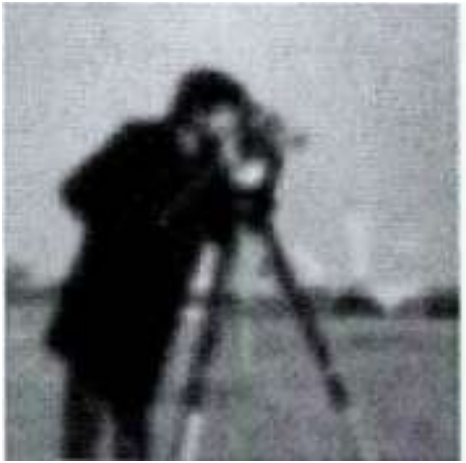

(a)

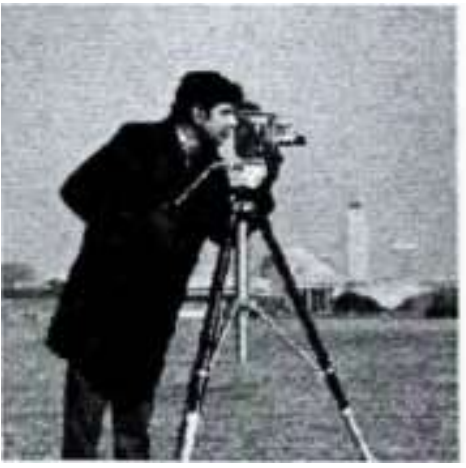

(c)

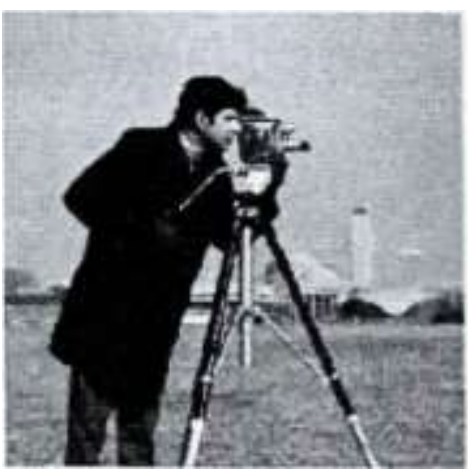

(e)

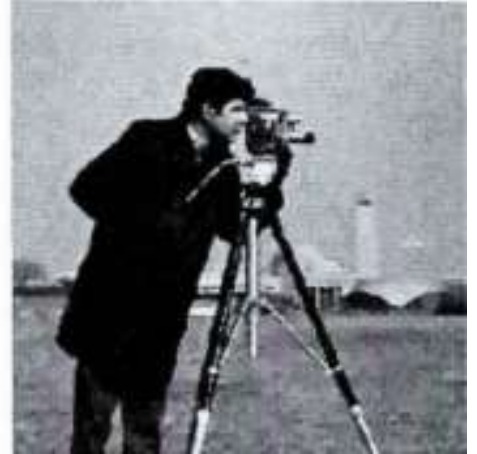

(b)

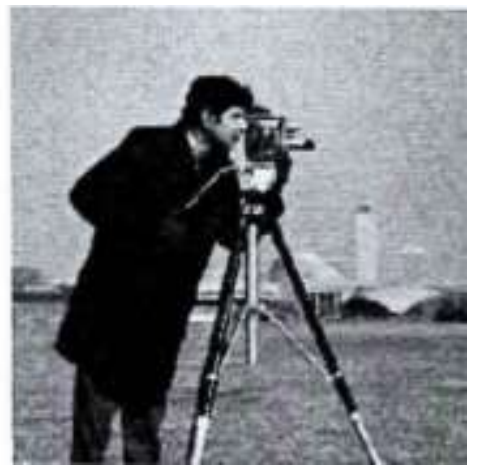

(d)

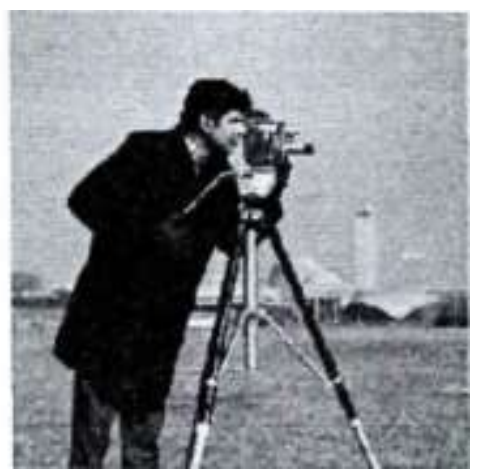

(f)

Fig. 2. Results of denoising obtained with Cameraman image: Result of ID algorithm (a); result of PM algorithm (b); result of TV algorithm (c); result of W algorithm (d); result of WT algorithm (e); result of new algorithm (f) 


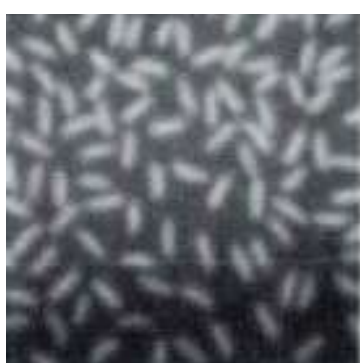

(a)

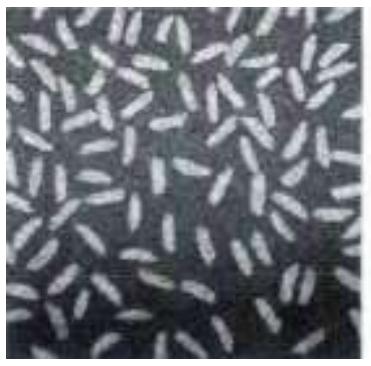

(c)

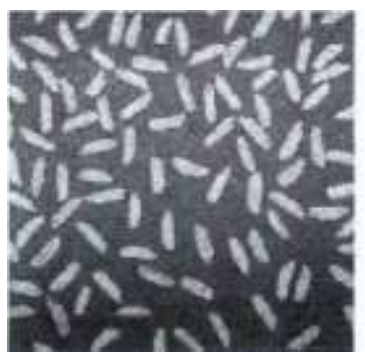

(e)

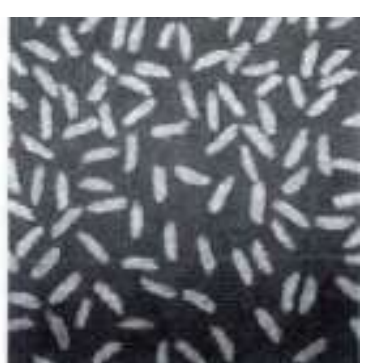

(b)

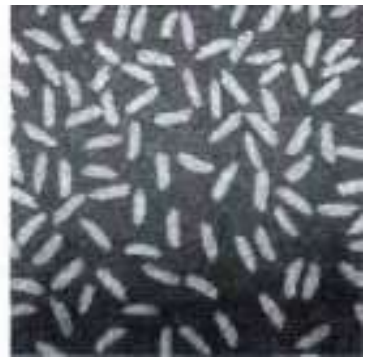

(d)

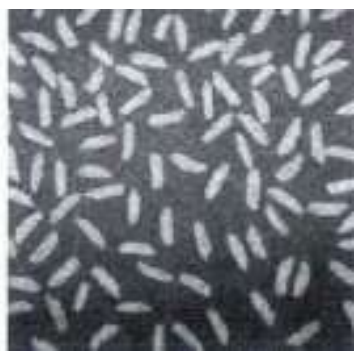

(f)

Fig. 3. Results of denoising obtained with Rice image: Result of ID algorithm (a); result of PM algorithm (b); result of TV algorithm (c); result of W algorithm (d); result of WT algorithm (e); result of new algorithm (f)

\section{Conclusion}

In this paper we propose a new approach for image denoising, based on the combination of PM model, isotropic diffusion model, and TV model. To emphasize the superiority of the proposed model, we have used the SSIM and PSNR as the subjective criterion.

Numerical experiments with different images show that our algorithm has the highest PSNR and SS1M as well as the best visual quality among the six algorithms. Experimental results confirm the high performance of the proposed model compared with some well-known algorithms. In a word, the new model outperforms the mentioned three well known algorithms in reducing the Gibbs-type artifacts, edges blurring, and the block effect, simultaneously. 
Acknowledgements: This work was financially supported by Chongqing Municipal Education Commission scientific and technological research project, China (No KJ1503209).

\section{References}

1. Li u, S., M. Shi, S. Hu, Y. Xia o. Synthetic Aperture Radar Image De-Noising Based on Shearlet Transform Using the Context-Based Model. - Physical Communication, 2014, pp. 245-248.

2. Gu p t a, V., V. Ch a u r a s i a, M. S h an d i l y a. Random-Valued Impulse Noise Removal Using Adaptive Dual Threshold Median Filter. - Journal of Visual Communication and Image Representation, 2014, pp. 5-8.

3. Y a n, Q., X. S u i. Random Noise Filtering Method Based on the Inter-Frame Registration. Infrared Physics and Technology, 2014, pp. 67-72.

4. Cai, Z., C. We i. A Tight Framelet Algorithm for Color Image De-Noising. - Procedia Engineering, 2011, pp. 24-30.

5. Zhang, D., X. Kang, J. W ang. A Novel Image De-Noising Method Based on Spherical Coordinates System. - EURASIP Journal on Advances in Signal Processing, 2012, pp. 201-214.

6. Nadernejad, E., S. Shar if zadeh, S. For chh a m mer. Using Anisotropic Diffusion Equations in Pixon Domain for Image De-Noising. - Signal, Image and Video Processing, 2013, pp. 76-82.

7. Seddik, H., S. Tebbini, E. B. B raiek. Smart Real Time Adaptive Gaussian Filter Supervised Neural Network for Efficient Gray Scale and RGB Image De-Noising. Intelligent Automation \& Soft Computing, 2014, pp. 203-211.

8. F a n g, J., Q. C a o. Total Variation Image De-Noising Bases on the Improved Sobel Operator. Journal of Multimedia, 2013, pp. 84-91.

9. Y i n, L., D. Che n, C. Li. Two-Dimensional Wavelet Transform De-Noising Algorithm in Collecting Intelligent Agriculture Image. - Journal of Software, 2013, pp. 84-89. 